\title{
ARTÍCULOS DE REVISIÓN \\ CONDUCTA SUICIDA: CONSIDERACIONES PRÁCTICAS PARA LA IMPLEMENTACIÓN DE LA TERAPIA COGNITIVA
}

\author{
SUICIDAL BEHAVIOR: \\ PRACTICAL ISSUES FOR COGNITIVE THERAPY DEVELOPMENT
}

\author{
RONALD TORO* \\ FUNDACIÓN SERÉ, BOGOTÁ - COLOMBIA \\ UNIVERSIDAD CATÓLICA DE COLOMBIA, BOGOTÁ - COLOMBIA
}

FECHA RECEPCIÓN: 4/12/2012・ FECHA ACEPTACIÓN: 13/2/2013

\section{Resumen}

En esta revisión documental, la Conducta Suicida (CS) se refiere a ideas, intentos y actos autoinfligidos de muerte, causada por cogniciones negativas autorreferentes (triada cognitiva), un procesamiento de información selectivo, rígido y generalizado, con sesgos confirmatorios y estilos de afrontamiento pobres. Puede tratarse con Terapia Cognitiva (TC), la cual ha demostrado efectividad en esta problemática, basada en la modificación de cogniciones negativas y estilos disfuncionales de afrontamiento, reducción de deserción, adherencia a servicios de salud y apoyo social. Sin embargo, existen dificultades en la demostración empírica de la efectividad de estas psicoterapias (tipo CBT), como la selección de la muestra (discriminación entre ideación, intento, comorbilidades, disponibilidad, reporte), grupos control, generalización de resultados, entre otros. Por tanto se analizan posibilidades metodológicas como clasificar los grupos según patología psiquiátrica o tipo de conducta suicida: intento, ideación y control, llevar enfoques de conductas de riesgo y desesperanza, y usar modelos estadísticos actualizados, entre otros. Se concluye recomendando la TC, aunque solicitando permanentes mejoras metodológicas.

Palabras clave: suicidio, terapia cognitiva, pensamiento, metodología.

\section{Abstract}

Suicidal Behavior (CS) refers to ideas, attempts and acts of self-inflicted death, caused by self-referential negative cognitions (cognitive triad), rigid and generalized selective information processing, confirmatory bias and poor coping profiles. It can be treated with cognitive therapy (CT), which has proven effective for this problem, based on the modification of negative cognitions and dysfunctional copings strategies, desertion reduction, adherence to health services and social support. However, there are many difficulties in the empirical demonstration of the effectiveness of these psychotherapies (CBT type) as the sample selection (discrimination between ideation, intent, comorbidities, availability, reporting), control groups, generalization of results, among others. Therefore we analyze methodological possibilities as classifying the groups according type of psychiatric illness or suicidal behavior: attempt, suicidal ideation and control approaches carry risk behavior and hopelessness, and use statistical models to date. It concludes recommending CT, but requesting permanent methodological improvements. Keywords: suicide, cognitive therapy, thinking, methodology.

* Psicólogo Especialista en Psicología Clínica, Candidato a Magíster en Psicología con énfasis en Psicología Clínica en la Universidad Católica de Colombia. Investigador de la Fundación SERÉ. La correspondencia debe ser enviada a la Carrera Séptima \# 17-01 oficina 840, Bogotá, Colombia. Email: tororonald@gmail.com 


\section{Introducción}

La Clasificación Internacional de Enfermedades CIE-10 (OMS, 1992), define al suicidio como una muerte por lesión autoinflingida, deliberadamente iniciado por la persona, intencional, como medio para obtener un resultado deseado, consciente y social, resultado de la interacción de factores biológicos, genéticos, psicológicos, sociológicos, culturales y ambientales (OMS, 2006), es decir, un hecho multicausal (Serrano, 2003), que requiere tener en cuenta diferentes consideraciones metodológicas al momento de implementar un plan de psicoterapia, tema que constituye el objetivo del presente trabajo de revisión.

Se requiere antes de iniciar, tener en cuenta la terminología asociada al suicidio. En primer lugar, al tratarse de un comportamiento altamente asociado con la capacidad suicida. Esta es la capacidad psicológica que adquiere la persona para tolerar dolor y/o temor a lo largo de una prolongada exposición a situaciones de difícil manejo, y como un importante indicador de probabilidad de muerte por el acto suicida (Smith, Cukrowicz, Poindexter, Hobson \& Cohen, 2010). El constructo de Conducta Suicida (CS) es de igual importancia, puesto que hace referencia a una amplia gama de conductas manifiestas en términos de ideas, amenazas, intentos y el suicidio consumado. La ideación suicida se caracteriza por planes y deseos constantes de cometer suicidio (Rosello \& Berrios, 2004), se refiere por tanto a pensamientos serios y persistentes acerca del deseo de matarse o morir (Barlow \& Durand, 2001; Rosello \& Berrios, 2004); el intento suicida es un acto autolesivo con cualquier grado de intención letal (Pérez-Olmos, Ibáñez-Pinilla, ReyesFigueroa, Atuesta-Fajardo \& Suárez-Díaz, 2008), y la amenaza suicida, es cuando la persona a pesar de tener los medios no logra su objetivo. Boldt (1989), agrega que se debe partir de tres supuestos básicos: es un acto voluntario, el individuo desea morir y percibe su situación como intolerable e irremediable.

Si bien, la población de mayor riesgo son los adolescentes y adultos jóvenes a nivel mundial (OMS, 2006; 2000), -la cual ha tenido un incremento significativo año tras año (García-Rábago, Sahagún-Flores, Ruiz-Gómez, Sánchez-Ureña, Tirado-Vargas \& GonzálezGámez, 2010)-, ha sido foco para el desarrollo de di- ferentes programas de prevención como es el caso del programa SUPRE (Suicide Prevention) (OMS, 2000), desarrollado con el objetivo de disminuir la conducta suicida. Actualmente es una de las tres principales causas de muerte entre personas de 15 a 35 años de edad, donde se cometen intentos cada tres segundos, con éxito cada 40 segundos y con un impacto en alrededor de seis personas, según reportes de la OMS (2000; 2006). En Colombia es una de las mayores problemáticas de salud pública según el informe del Instituto de Medicina Legal y Ciencias Forenses (2011), más frecuente en hombres, con marcada tendencia al incremento en los últimos sesenta años, según la OMS (2007).

En el estudio realizado por Pérez-Olmos et ál. (2008), se encontró que en población bogotana, la persistencia de ideación suicida fue mayor en individuos cuyo perfil estaba caracterizado por la severidad de la disfunción psiquiátrica crónica y la dimensión familiar y social, contando con 31 años o más, sin ocupación con función social, conflictos no resueltos o empeorados, disfunción familiar, con una red de apoyo débil, enfermedad médica, intentos suicidas previos o uno de alta letalidad, haber sido tratado con psicofármacos con o sin psicoterapia, y hospitalizado en alguna unidad de salud mental por trastorno depresivo grave o patología psiquiátrica activa.

Finalmente, en el estudio realizado por Posada, Rodríguez, Duque y Garzón (2007) frente al Estudio de Salud Mental (2003) en Colombia, la prevalencia y factores de riesgo de comportamientos suicidas encontró que la cohorte de 35 a 45 años de edad, después del primer empleo, con sexo femenino, ser joven y tener desventaja social, además del haber experimentado una edad de inicio de intentos suicidas temprana, fueron reportados como altos factores de riesgo en Colombia para comportamientos relacionados con el suicidio.

En cuanto a las posibles causas de la aparición y mantenimiento de la CS, desde una perspectiva neurobiológica y psicológica, es necesario estudiarla desde dos grandes categorías de riesgo suicida: la primera en cuanto a un déficit en control de impulsos y la segunda en cuanto a la propensión al dolor psicológico intenso. Si bien, cada una de estas categorías está sustentada en variados factores genéticos y neurobiológicos específicos (v. g. Joiner, Brown \& Wingate, 2005), que llegan a afectar el sistema serotoninérgico, entre otros. 
Otra de las causas está en los patrones cognitivos de procesamiento de información, creencias disfuncionales y mecanismos de preservación esquemática (Beck, Rush, Shaw \& Emery, 1979; Freeman \& Reinecke, 1995; Henriques, Beck \& Brown, 2003; Rush \& Beck, 1978). El modelo teórico de la Terapia Cognitiva (TC), parte del supuesto básico de que las personas suelen padecer psicopatologías no por las situaciones en sí, sino por la interpretación que suelen hacer de estas (Beck \& Freeman, 1990; Beck, Rush, Shaw \& Emery, 1979). En el caso de la CS, el modelo afirma que es una «expresión extrema del deseo de escape de lo que parece ser un problema irresoluble o una situación insoportable» (Rush \& Beck, 1978; p. 202) y se deriva de tres componentes de la triada cognitiva negativa: yo defectuoso, un mundo agresivo y despiadado, y un futuro desolador y desesperanzado. A lo anterior se agregan ciertos errores cognitivos dominantes y unas estructuras cognitivas relativamente estables denominados esquemas disfuncionales activos (Beck et ál., 1979; Rush \& Beck, 1978), por tanto el objetivo de la evaluación en esta terapia, inicialmente se centra en la identificación de pensamientos automáticos, distorsiones cognitivas y creencias centrales que se activan inmediatamente antes del intento de suicidio (Beck et ál., 1979).

El modelo cognitivo básico de la CS inicia con una explicación acerca de su etiología y mantenimiento, que como consecuencia de la activación de esquemas disfuncionales, las personas desarrollan una triada cognitiva depresiva: una visión negativa de sí mismos, del mundo y del futuro, sobredimensionada esta última en pacientes con CS, cuyos patrones cognitivos están relacionados con la múltiple sintomatología del proceso suicida y el síndrome depresivo, como el caso de las personas con abulia, la cual es una disminución o pérdida de la voluntad hacia acciones específicas (propia de estos pacientes), con elevada desesperanza y pesimismo, ignorando a su vez la información que desconfirma su información esquemática activada. Es decir, filtran aquella información que se ajuste al esquema disfuncional, sobre estiman las dificultades que se generan negando las habilidades propias y enfocando permanentemente la posibilidad de fracaso. Al paso del despliegue del proceso suicida, los pensamientos automáticos negativos se tornan más reiterativos, generan un malestar psicológico que desemboca en un estado de ánimo melancólico, retroalimentado en un permanente círculo vicioso (Beck et ál., 1979; Beck, Steer, Kovacs, \& Garrison, 1985; Freeman \& Reinecke, 1995; Rush \& Beck, 1978).

Por otra parte, la intervención cognitiva para la conducta suicida nació en el modelo cognitivo de los trastornos emocionales (Beck, 1976), en depresión en particular (Beck, 1963; 1964; 1967; Beck et ál., 1979), así como la extensa literatura que une la desesperanza con la CS (Abramson, Metalsky \& Alloy, 1989; Abramson, Metalsky, Alloy, Joiner \& Sandin, 1997; Beck, Steer, Kovacs \& Garrison, 1985; Rush \& Beck, 1978).

Por ejemplo, en el trabajo de Henriques, Beck y Brown (2003), resaltan acerca de la intervención cognitiva de la CS que la característica central es la identificación de los pensamientos automáticos, junto con las creencias nucleares que se activaron justo antes del intento suicida, y luego, con los componentes cognitivos particulares identificados, algunas de las estrategias cognitivo-conductuales se aplican para desarrollar formas más adaptativas de pensar acerca de sus situaciones de un modo más funcional y de responder durante periodos de estrés emocional agudo.

Por tanto, el foco de la terapia cognitiva TC es reducir la conducta suicida con los siguientes elementos claves: a) el desarrollo de medios eficaces para hacer frente a la desesperanza y a la conducta objetosuicida, b) involucrar a los pacientes rápidamente en el tratamiento para reducir la deserción, c) aumentar el uso adaptativo de los servicios de salud, y d) aumentar el uso adaptativo del apoyo o soporte social. En resumen, la estructura de la TC para Conducta Suicida, debe contar con una serie de componentes que además del adecuado enganche terapéutico, están la enseñanza en solución de problemas, la conceptualización de caso clínico, la reestructuración cognitiva y conductual, un «kit de esperanza» y uno de afrontamiento, reducir la impulsividad y ampliar redes de soporte social (Brown, Ten, Henriques, Xie, Hollander \& Beck, 2005; Henriques, Beck \& Brown, 2003). En la tabla 1 se presenta un resumen del tratamiento cognitivo conductual, según la propuesta de Henriques, Beck y Brown (2003) y Brown, Ten, Henriques, Xie, Hollander y Beck (2005). 


\section{Metodología para la terapia cognitiva en conducta suicida}

El Task Force (American Psychological Association-APA, división 12, 2005), ha buscado diseminar los procedimientos para la elaboración de tratamientos efectivos y eficaces en el manejo de intervenciones en psicología desde hace varios años, con publicaciones clásicas como la desarrollada por Chambless y Hollon (1998), en la cual detallaron la forma en la cual deberán desarrollarse estudios controlados para demostrar efectividad, o al menos la futura efectividad de los procedimientos tomando como variables dependientes los efectos de los tratamientos, basados en interrogantes que abarcan al menos tres aspectos claves: a) ¿demostró ser beneficioso en un estudio controlado?, b) ¿es útil en ambientes clínicamente controlados u otros?, y c) ¿su relación costo-beneficio lo hace un procedimiento viable?

Además, una de las características sugeridas en estos estudios es la posibilidad de replicarlo, tema aún controversial al demostrar la eficacia de un tratamiento debido a las dificultades inherentes a las replicaciones en los procesos psicoterapéuticos (Chambless \& Hollon, 1998), como son los tamaños de las muestras, las variaciones entre los participantes, y el caso especial de la CS cuando los diseños muestrales cambian de acuerdo a la disponibilidad y la frecuencia de intentos suicidas reportados y asequibles a los procedimientos psicoterapéuticos (Pérez-Olmos et ál., 2008).

Tabla 1. Fases del tratamiento cognitivo conductual para la conducta suicida por sesiones de intervención (Brown, Ten, Henriques, Xie, Hollander \& Beck, 2005; Henriques, Beck \& Brown, 2003).

\begin{tabular}{|c|c|c|c|c|c|c|c|}
\hline Sesiones & & & tividades psi & coterapéutic & cas & & \\
\hline $\begin{array}{l}\text { 1-3 Fase } \\
\text { primaria } \\
\text { del tra- } \\
\text { tamiento }\end{array}$ & $\begin{array}{l}\text { Enganchar } \\
\text { el paciente } \\
\text { al tra- } \\
\text { tamiento. }\end{array}$ & $\begin{array}{ll}\text { Pedir que } & \text { Atender el } \\
\text { «cuente su } & \text { riesgo de la } \\
\text { propia his- } & \text { conducta } \\
\text { toria» que } & \text { suicida del } \\
\text { lo llevó al } & \text { paciente. } \\
\text { intento de } & \\
\text { suicidio. } & \end{array}$ & $\begin{array}{l}\text { Desarrollar } \\
\text { un plan se- } \\
\text { guro para la } \\
\text { emergencia } \\
\text { suicida. }\end{array}$ & $\begin{array}{l}\text { Orientar } \\
\text { al paciente } \\
\text { al modelo } \\
\text { cognitivo } \\
\text { (psico- } \\
\text { educación). }\end{array}$ & $\begin{array}{l}\text { Iniciar el } \\
\text { desarro- } \\
\text { llo de la } \\
\text { concep- } \\
\text { tualización } \\
\text { del intento } \\
\text { suicida. }\end{array}$ & $\begin{array}{l}\text { Enseñar } \\
\text { solución de } \\
\text { problemas } \\
\text { (desarrollar } \\
\text { lista de } \\
\text { problemas } \\
\text { y metas). }\end{array}$ & $\begin{array}{l}\text { Recuperar } \\
\text { el sentido } \\
\text { de esperanza } \\
\text { del paciente. }\end{array}$ \\
\hline $\begin{array}{l}\text { 4-7 Fase } \\
\text { intermedia } \\
\text { del tra- } \\
\text { tamiento }\end{array}$ & $\begin{array}{l}\text { Desarrollar } \\
\text { nuevas he- } \\
\text { rramientas } \\
\text { para reac- } \\
\text { cionar a las } \\
\text { situaciones } \\
\text { estresoras, } \\
\text { con base en } \\
\text { la reestruc- } \\
\text { turación } \\
\text { cognitiva y } \\
\text { el cambio } \\
\text { conductual. }\end{array}$ & $\begin{array}{l}\text { Identificar y modificar } \\
\text { las creencias centrales } \\
\text { asociadas al intento sui- } \\
\text { cida y sus estrategias de } \\
\text { afrontamiento acompa- } \\
\text { ñantes } \\
\text { Identificar y modificar } \\
\text { las creencias centrales } \\
\text { asociadas al intento sui- } \\
\text { cida y sus estrategias de } \\
\text { afrontamiento acompa- } \\
\text { ñantes }\end{array}$ & $\begin{array}{l}\text { Reestructu- } \\
\text { rar creencias } \\
\text { desadapta- } \\
\text { tivas y pen- } \\
\text { samientos } \\
\text { automáticos } \\
\text { enseñando } \\
\text { la relación } \\
\text { pensa- } \\
\text { mientos- } \\
\text { emociones- } \\
\text { conductas }\end{array}$ & $\begin{array}{l}\text { Enseñar al } \\
\text { paciente } \\
\text { técnicas que } \\
\text { propicien } \\
\text { respuestas } \\
\text { adaptativas } \\
\text { frente al } \\
\text { pensa- } \\
\text { miento } \\
\text { disfuncional } \\
\text { (ej. tarjetas } \\
\text { de afron- } \\
\text { tamiento: } \\
\text { pensamien- } \\
\text { to suicida } \\
\text { vs. Respues- } \\
\text { ta alterna- } \\
\text { tiva. }\end{array}$ & $\begin{array}{l}\text { Construir un } \\
\text { Kit de Espe- } \\
\text { ranza para } \\
\text { los casos de } \\
\text { crisis, el cual } \\
\text { contiene una } \\
\text { lista de las } \\
\text { razones por } \\
\text { las cuales vi- } \\
\text { vir. Puede in- } \\
\text { cluir también } \\
\text { fotografías } \\
\text { y elementos } \\
\text { significativos, } \\
\text { ubicado en } \\
\text { un lugar } \\
\text { estratégico } \\
\text { de acceso en } \\
\text { la casa }\end{array}$ & $\begin{array}{l}\text { Construir } \\
\text { herra- } \\
\text { mientas } \\
\text { de afron- } \\
\text { tamiento } \\
\text { afectivas } \\
\text { para el al- } \\
\text { cance de las } \\
\text { metas del } \\
\text { paciente y } \\
\text { aumentar la } \\
\text { tolerancia } \\
\text { al malestar } \\
\text { psicológico }\end{array}$ & $\begin{array}{l}\text { Aumentar la } \\
\text { regulación } \\
\text { emocional } \\
\text { sin acudir } \\
\text { a la deses- } \\
\text { peración } \\
\text { y desespe- } \\
\text { ranza. }\end{array}$ \\
\hline
\end{tabular}




\begin{tabular}{|c|c|c|c|c|c|c|c|c|}
\hline Sesiones & \multicolumn{8}{|c|}{ Actividades psicoterapéuticas } \\
\hline $\begin{array}{l}\text { 4-7 Fase } \\
\text { intermedia } \\
\text { del tra- } \\
\text { tamiento }\end{array}$ & $\begin{array}{l}\text { Propiciar el } \\
\text { Control de } \\
\text { la impulsi- } \\
\text { vidad asu- } \\
\text { miendo a } \\
\text { los pacien- } \\
\text { tes como } \\
\text { «procasti- } \\
\text { nos» alrede- } \\
\text { dor de sus } \\
\text { impulsos } \\
\text { suicidas, } \\
\text { con un } \\
\text { diagrama de } \\
\text { gráficos del } \\
\text { estado de } \\
\text { ánimo y las } \\
\text { tendencias } \\
\text { suicidas a } \\
\text { través del } \\
\text { tiempo. }\end{array}$ & $\begin{array}{l}\text { Incre- } \\
\text { mentar el } \\
\text { soporte } \\
\text { social. }\end{array}$ & $\begin{array}{l}\text { Implemen- } \\
\text { tar gradual- } \\
\text { mente } \\
\text { una red } \\
\text { adaptativa } \\
\text { de sopor- } \\
\text { te social } \\
\text { accesible. }\end{array}$ & $\begin{array}{l}\text { Incrementar } \\
\text { el acompa- } \\
\text { ñamiento de } \\
\text { los servicios } \\
\text { sociales y de } \\
\text { la salud. }\end{array}$ & $\begin{array}{l}\text { Entrenar } \\
\text { Control de } \\
\text { la impul- } \\
\text { sividad } \\
\text { basado en } \\
\text { el efecto de } \\
\text { la procasti- } \\
\text { nación en la } \\
\text { aparición de } \\
\text { los impul- } \\
\text { sos suici- } \\
\text { das. Usar } \\
\text { diagrama } \\
\text { de gráficos } \\
\text { del estado } \\
\text { de ánimo y } \\
\text { tendencias } \\
\text { suicidas en } \\
\text { una unidad } \\
\text { de tiempo. }\end{array}$ & $\begin{array}{l}\text { Incrementar } \\
\text { el soporte } \\
\text { social. }\end{array}$ & $\begin{array}{l}\text { Implemen- } \\
\text { tar gra- } \\
\text { dualmente } \\
\text { una red } \\
\text { adaptativa } \\
\text { de soporte } \\
\text { social acce- } \\
\text { sible. }\end{array}$ & $\begin{array}{l}\text { Incrementar } \\
\text { el acompa- } \\
\text { ñamiento de } \\
\text { los servicios } \\
\text { sociales y de } \\
\text { la salud. }\end{array}$ \\
\hline $\begin{array}{l}\text { Finales del } \\
\text { tratamiento }\end{array}$ & \multicolumn{8}{|c|}{$\begin{array}{l}\text { Se propicia el incremento de las herramientas cognitivo-conductuales con tareas de prevención de recaídas } \\
\text { desde el afrontamiento del pensamiento suicida. Usar una guía imaginaría de prevención de recaídas (RTP, } \\
\text { Relapse Prevention Task) como cierre de tratamiento. }\end{array}$} \\
\hline
\end{tabular}

Fuente: elaboración propia.

Otra de las dificultades a mencionar está el establecer las diferencias claras entre el tratamiento para la depresión unipolar siguiendo el modelo cognitivo (Beck et ál.,1979), con el cual, pese a su soporte empírico en permanente revisión (Beck, 1995; Clark, Beck \& Alford, 1999), no se han definido con claridad los procedimientos específicos para la CS, ya que han sido adaptaciones de la TC para la depresión unipolar (Brown, Ten, Henriques, Xie, Hollander \& Beck, 2005; Chachamovich, Stefanello, Botega \& Turecki, 2009), el subtipo de la depresión por desesperanza (Abramson, Metalsky \& Alloy, 1989; Abramson, Metalsky, Alloy, Joiner \& Sandin, 1997; Clark, Beck \& Alford, 1999), manejo de crisis (Dedić, 2012), e inclusive el desarrollo de manuales para pacientes que incluyen casos con riesgo suicida (v. g. Greenberger \& Padesky, 1998) y números completos de publicaciones especializadas de la salud mental (v. g. Sakinofsky, 2007; Szumilas \& Kutcher, 2011).
Una forma de manejo metodológico a este inconveniente, es establecer diferencias claras entre los grupos participantes, tal como se discriminan los pacientes en el estudio llevado a cabo por Marquart, Overholser y Peak (2009), donde separaron los participantes de acuerdo al diagnóstico previo de depresión mayor, distimia, con ideación suicida e intentos suicidas crónicos, sin criterios de trastorno bipolar, esquizofrenia ni ningún otro problema orgánico cerebral. Aunque varios cumplían criterio de abuso de sustancias psicoactivas, se pueden realizar sencillamente agrupaciones control, intento suicida, e ideación suicida como el realizado por Smith, Cukrowicz, Poindexter, Hobson y Cohen (2010); o el obtener una muestra con pacientes en elevado riesgo con CS activa, es decir, pacientes con intentos de muerte recientes, que han sido atendidos en unidades de salud prioritaria y luego se les ha solicitado psicoterapia, tal como lo hicieron en un hospital universitario, según el trabajo publicado por 
Brown, Ten, Henriques, Xie, Hollander y Beck (2005). De igual forma, incluir en los diseños muestrales aquellos participantes que han elaborado contratos de «no suicidio» o «convenios de no suicidio», en procesos para casos de elevado riesgo (v. g. Edwards \& Harries, 2007), y que expresen deseo de recibir ayuda profesional (v. g. Dyregrov, 2009).

Por otra parte, es necesario enfocarse en aquellos factores de riesgo que conllevan a la CS para poder modificarlos, en la cual se deba elaborar una lista de seguimiento y observación continua (Chachamovich, Stefanello, Botega \& Turecki, 2009), no solamente centrada en las conductas de riesgo, sino las cogniciones que hacen a la persona vulnerable a la CS como las relacionadas con desesperanza (Beck, Steer, Kovacs, \& Garrison, 1985), teniendo claros previamente los riesgos del generalizar evaluaciones en pacientes con elevada vulnerabilidad psicopatológica (v. g. Barlow, Waegemakers, Chugh, Rawlinson, 2010); consideraciones evidentes en revisiones como la hecha por Mathews y MacLeod (2005) o el trabajo exploratorio de Vargas-Mendoza (2010) con pacientes sometidos a quimioterapia.

Igualmente, el usar herramientas metodológicas actualizadas para el análisis molecular de los elementos claves del tratamiento de la CS con TC, como los modelos de análisis de ecuaciones estructurales (Chachamovich, Stefanello, Botega \& Turecki, 2009), estudios basados en comparación de medias pretest, postest y seguimiento (Momeni, Moshtagh \& Purshahbaz, 2010), estimativos como los de probabilidad de supervivencia de Kaplan-Meier (1958), estudios correlacionales simples (v. g. Roselló \& Berríos, 2004), análisis de varianza de una vía (v. g. Marquart, Overholser \& Peak, 2009), análisis de regresión para modelos predictivos como algunos estudios de vulnerabilidad (Sutton et ál., 2010), y el uso de medidas psicofisiológicas (potenciales evocados) como las utilizadas con imágenes relacionadas con ideas suicidas, siguiendo el trabajo de Smith et ál. (2010); además teniendo en cuenta la necesidad de utilizar instrumentos adaptados a la cultura y el idioma local, como se evidencia en el trabajo de Joe, Woolley, Brown, GhahramanlouHolloway y Beck (2008), acerca de las propiedades psicométricas del BDI-II en Sudáfrica y su relación con la CS.

Un importante aspecto a resaltar, siguiendo la revisión de Daigle, Pouliot, Chagnon, Greenfield \& Misha- ra (2010), ha sido desarrollar estudios controlados con grupos placebos en CBT para CS, en el cual compararon -incluida también la terapia psicodinámica breve-, el uso de una serie de estrategias de prevención activa de recaídas como llamadas periódicas, visitas domiciliarias, tarjetas de emergencia, hospitalizaciones y programas con una intensidad de atención individualizada, dos veces por semana (cabe destacar que solo uno de estos cuatro métodos suelen reportarlo como eficaz), aunque reportaron persistencia en dificultades de diferenciación de género, medidas de las variables dependientes en estos procedimientos e incluso la misma solidez de los programas de apoyo comunitario locales, independientemente de los sistemas de psicoterapia comparados.

Además, es recomendable no dejar de lado aquellas revisiones sistemáticas de investigaciones actualizadas que se relacionen con la CS, como la elaborada por Sakinofsky (2007), con respecto al uso de tratamientos farmacológicos combinados para CS y depresión, y su dudosa efectividad de los medicamentos antidepresivos desde la misma prescripción.

Un aspecto final, no menos válido, es lo que corresponde al enganche terapéutico (asociado a la asistencia, compromiso y seguimiento de reglas) y su directa relación con la relación terapéutica (asociada a la calidad de la comunicación, alianza, confianza, simpatía y empatía), que han sido objeto de estudios en diferentes trastornos en la búsqueda de resultados satisfactorios (Dingle, Gleadhill \& Baker, 2008), aunque sea en procedimientos breves (v. g. Fleischmann et ál., 2008) como el estudio llevado a cabo por Constantino, Manber, Ong, Kuo y Arnow (2007), donde presentaron la CBT para remisión del insomnio y su relación con expectativas hacia la psicoterapia y percepción confrontativa del terapeuta.

De igual forma, identificar la adherencia como en el estudio de Esposito-Smythers, Spirito, Uth y LaChance (2006), con adolescentes con intentos suicidas y consumo de alcohol, donde primó la fuerte relación terapéutica y una percepción de escasos problemas con el tratamiento efectivo por parte de los adolescentes, cuando puntuaron con listas de chequeo la adherencia al tratamiento por sesión en un 80\%; además, es indispensable tener en cuenta variables sociales como el estilo de apego, problemas interpersonales y apoyo social disponible (Ilardi \& Kaslow, 2009). 
Una sugerencia frente a este aspecto es tomar una línea de base con medidas objetivas del nivel de insight, funcionamiento social y niveles de alianza grupal, y una metodología de investigación basada en procedimientos lineales jerárquicos para poder predecir el enganche terapéutico, como lo plantearon Johnson, Penn, Bauer, Meyer y Evans (2008), en su estudio con pacientes esquizofrénicos y terapia grupal; o usar medidas pre-inter-pos tratamiento, como forma de seguimiento del proceso en promedio de 10 sesiones, como el trabajo elaborado por Chiu, McLeod, Har y Wood (2009), con niños ansiosos.

\section{Conclusiones}

Es indispensable resaltar aquellas limitaciones metodológicas con respecto al uso de grupos comparativos (sobrellevando las conocidas dificultades con la CS específicamente mencionadas), puesto que se evidencian escasas diferencias entre grupos de control sin CBT (en espera) y con CBT (v. g. Hetrick, Purcell, Garner \& Parslow, 2010), aunque sí se reportan diferencias entre CBT y otras psicoterapias para la CS, por lo menos en adultos jóvenes con elevado riesgo suicida (v. g. Robinson, Hetrick \& Martin, 2011), lo que lleva en últimas a cuestionarse acerca de la efectividad de los tratamientos y las variables que deberán ser controladas con mayor rigurosidad en futuros estudios al respecto, principalmente cuando se trate de fortalecer psicoterapias como la TC, o el uso de la CBT en la CS y trastornos concomitantes, y así llegar a establecer esta interminable lista de las consideraciones breves, a tener en cuenta a la hora de implementar la TC, procedimiento en permanente revisión empírica.

\section{Referencias}

Abramson, L. Y., Metalsky, G. I., \& Alloy, L. B. (1989). Hopelessness Depression: A Theory-Based Subtype of Depression. Psychological Review, 96, 358-372. Abramson, L. Y., Metalsky, G. I., Alloy, L. B., Joiner, Th. E. \& Sandin, B. (1997). Teoría de la depresión por desesperanza: Aportaciones recientes. Revista de Psicopatología y Psicología Clínica, 2 (3), 211-222.

American Psychological Association, APA (2005). Report of the 2005 Presidential Task Force on EvidenceBased Practice. Document received by the Ameri- can Psychological Association (APA) Council of Representatives during its meeting of August on 2005.

Barlow, C. A., Waegemakers Schiff, J., Chugh, U., Rawlinson, D., Hides, E. \& Leith, J. (2010). An Evaluation of a Suicide Bereavement Peer Support Program. Death Studies, 34 (10), 915-930. doi:10.1080/07481181003761435.

Barlow, D. \& Durand, V. M. (2001). Psicología anormal: un enfoque integral. México: Thompson.

Beck, A.T. (1963). Thinking and Depression: 1. Idiosyncratic Content and Cognitive Distortions. Archives of General Psychiatry, 9, 36-45.

Beck, A. T. (1964). Thinking and Depression. 2. Theory and Therapy. Archives of General Psychiatry, 10, 561571.

Beck, A. T. (1967). Depression: Clinical, Experimental, and Theoretical Aspects. New York: Harper and Row.

Beck, A. T. (1976). Cognitive Therapy of the Emotional Disorders. New York: New American Library.

Beck, A. T. (1995). Terapia cognitiva: pasado, presente y futuro. En M. Mahoney (comp.), Psicoterapias cognitivas y constructivistas. Teoría, investigación y práctica (pp. 49-61). Bilbao: Desclée de Brouwer.

Beck, A. T. \& Freeman, A. (1990). Cognitive Therapy of Personality Disorders. New York: Guilford.

Beck, A. T., Rush, A. J., Shaw, B. F. \& Emery, G. (1979). Cognitive Therapy of Depression. Nueva York: Guilford Press.

Beck, A. T., Steer, R. A., Kovacs, M. \& Garrison, B. (1985). Hopelessness and Eventual Suicide: A 10 Year Prospective Study of Patients Hospitalized with Suicidal Ideation. American Journal of Psychiatry, 42, 559-563.

Boldt, M. (1989). Defining Suicide. Implications for Suicidal Behavior and for Suicide Prevention. En Diekstra, R., Maris, R., Platt, S., Schmidtke, A. \& Sonneck, G. (Comp.) Suicide and its Prevention: the Role of Attitude and Imitation. World Health Organization. Netherlands: E. J. Brill.

Brown, G. K., Ten, Th., Henriques, G. R., Xie, S. X., Hollander, J. E. \& Beck, A. T. (2005). Cognitive Therapy for the Prevention of Suicide Attempts A Randomized Controlled Trial. Journal of the American Medical Association, 294, 563-570. 
Chachamovich, E., Stefanello, S., Botega, N. \& Turecki, G. (2009). Which are the Recent Clinical Findings Regarding the Association Between Depression and Suicide? Revista Brasileira de Psiquiatría, 31 (1), 18-25.

Chambless, D. L. \& Hollon, S. D. (1998). Defining Empirically Supported Therapies. Journal of Consulting and Clinical Psychology, 66 (1), 7-18.

Chiu, A., McLeod, B., Har, K. \& Wood, J. (2009). Childtherapist Alliance and Clinical Outcomes in Cognitive Behavioral Therapy for Child Anxiety Disorders. Journal of Child Psychology And Psychiatry, and Allied Disciplines, 50 (6), 751-758.

Constantino, M. J., Manber, R., Ong, J., Kuo, T. F., Huang, J. S., \& Arnow, B. A. (2007). Patient Expectations and Therapeutic Alliance as Predictors of Outcome in Group Cognitive-Behavioral Therapy for Insomnia. Behavioral Sleep Medicine, 5 (3), 210-228. doi:10.1080/15402000701263932.

Daigle, M. S., Pouliot, L. P., Chagnon, F., Greenfield, B. \& Mishara, B. (2010). Suicide Attempts: Prevention of Repetition. Canadian Journal of Psychiatry, 56 (10), 621-629.

Dedić, G. (2012). Model of Psychotherapeutic Crisis Intervention Following Suicide Attempt. Vojnosanitetski Pregled. Military-Medical And Pharmaceutical Review, 69 (7), 610-615.

Dingle, G.A., Gleadhill, L. \& Baker, F.A. (2008). Can music therapy engage patients in group cognitive behavior therapy for substance abuse treatment? Drug and Alcohol Review, 27, 190-196.

Dyregrov, K. (2009). How do the Young Suicide Survivors wish to be Met by Psychologists? A User Study. Omega, 59 (3), 221-238.

Edwards, S. \& Harries, M. (2007). No-suicide Contracts and No-suicide Agreements: a Controversial Life. Australasian Psychiatry: Bulletin of Royal Australian and New Zealand College of Psychiatrists, 15 (6), 484-489.

Esposito-Smythers, C., Spirito, A., Uth, R. \& LaChance, H. (2006). Cognitive Behavioral Treatment for Suicidal Alcohol Abusing Adolescents: Development and Pilot Testing. The American Journal on Addictions, 15, 126-130.

Fleischmann, A., Bertolote, J. M., Wasserman, D., De Leo, D., Bolhari, J., Botega, N. J. et ál. (2008).
Effectiveness of Brief Intervention and Contact for Suicide Attempters: a Randomized Controlled Trial in Five Countries. Bulletin of the World Health Organization, 86 (9), 703-709.

Freeman, A. \& Reinecke, M. (1995). Terapia cognitiva aplicada a la conducta suicida. Bilbao: Desclee de Brouwer.

García-Rábago, H., Sahagún-Flores, J. E., Ruiz-Gómez, A., Sánchez-Ureña, G. M., Tirado-Vargas, J. C. \& González-Gámez, J. G. (2010). Factores de riesgo, asociados a intento de suicidio, comparando factores de alta y baja letalidad. Revista de salud pública, 12 (5), 713-721.

Greenberger, D. \& Padesky, C. A. (1998). El control de tu estado de ánimo: Manual de tratamiento de terapia cognitiva para usuarios. Barcelona: Paidós. (Edición original en inglés Mind over Mood: Change How You Feel by Changing the Way You Think, (1995). New York: The Guilford Press).

Henriques, G., Beck A.T. \& Brown, G. (2003). Cognitive Therapy for Adolescent and Young Adult Suicide Attempters. The American Behavioral Scientist, 46 (9), 1258-1268.

Hetrick, S. E., Purcell, R., Garner, B. \& Parslow, R. (2010). Combined Pharmacotherapy and Psychological Therapies for Posttraumatic Stress Disorder (PTSD). Cochrane Database of Systematic Reviews, 7 (CD0073 16). DOI: 10.1002/14651858. CD007316.pub2.

Ilardi, D. L. \& Kaslow, N. J. (2009). Social Difficulties Influence Group Psychotherapy Adherence in Abused, Suicidal African American Women. Journal of Clinical Psychology, 65 (12), 1300-1311.

Instituto de Medicina Legal y Ciencias Forenses (2011). Boletín estadístico mensual (marzo de 2011). Centro de Referencia Nacional sobre Violencia -CRNV. Base: Sistema de Información Red de Desaparecidos y Cadáveres-SIRDEC. Disponible en www.medicinalegal. gov.co

Joe, S., Woolley, M. E., Brown, G. K., GhahramanlouHolloway, M. \& Beck, A. T. (2008). Psychometric Properties of the Beck Depression Inventory-II in Low-Income, African American Suicide Attempters. Journal of Personality Assessment, 90 (5), 521-523. 
Johnson, D. P., Penn, D. L., Bauer, D. J., Meyer, P. \& Evans, E. (2008). Predictors of the Therapeutic Alliance in Group Therapy for Individuals with Treatment-Resistant Auditory Hallucinations. British Journal of Clinical Psychology, 47(2), 171-183. doi:10.1348/014466507X241604.

Joiner, Th. E., Brown, J. S. \& Wingate, L. R. (2005). The Psychology and Neurobiology of Suicidal Behavior. Annual Review of Psychology, 56, 287-314.

Kaplan, E. L. \& Meier, P. (1958). Non-Parametric Estimation from Incomplete Observations. Journal of American Statistics Association, 3, 457-481.

Marquart, A. L., Overholser, J. C. \& Peak, N. J. (2009). Mood Regulation Beliefs in Depressed Psychiatric Inpatients: Examining Affect, Behavior, Cognitive, and Social Strategies. International Journal of Psychiatry in Clinical Practice, 13, 265-272.

Mathews, A. \& MacLeod, C. (2005). Cognitive Vulnerability to Emotional Disorders. Annual Review of Clinical Psychology, 1, 167-195.

Momeni, F., Moshtagh, N. \& Purshahbaz, A. (2010). Effectiveness of Cognitive-Behavioral Group Therapy on Craving, Depression, Anxiety Among the Opiate Abusers Under MMT. Iranian Rehabilitation Journal, 8 (11), 19-23.

Organización Mundial de la Salud. (1992). CIE 10. Trastornos mentales y del comportamiento. Décima revisión de la Clasificación Internacional de las Enfermedades. Descripciones Clínicas y pautas para el diagnóstico. Ginebra: OMS.

Organización Mundial de la Salud. (2000). Prevención del suicidio. Un instrumento para trabajadores de atención primaria de salud. Documento no formal de la Organización Mundial de la Salud, disponible en www. who.int/mental_health/media/primaryhealthcare_workers_spanish.pdf

Organización Mundial de la Salud. (2000). Preventing Suicide: a Resource for Teachers and Other School Staff Mental and Behavioural Disorders. Ginebra (Suiza): Mental and Behavioural Disorders. Department of Mental Health Of World Health Organization.

Organización Mundial de la Salud. (2006). Prevención del suicidio recurso para consejeros. Departamento de Salud Mental y Abuso de Sustancias Trastornos Mentales y Cerebrales. Ginebra: OMS.
Organización Mundial de la Salud. (2007). Suicide Rates (per 100,000), by Gender, Colombia, 1950-2007. Consultado en Febrero de 2013. Disponible en www.who.int/mental_health/media/colo.pdf

Pérez-Olmos, I., Ibáñez-Pinilla, M., Reyes-Figueroa, J., Atuesta-Fajardo, J. \& Suárez-Díaz, M. (2008). Factores asociados al intento suicida e ideación suicida persistente en un centro de atención primaria. Bogotá, 2004-2006. Revista de Salud Pública, 10, 3, 374-385.

Posada, J., Rodríguez, M., Duque, P. \& Garzón, A. (2007). Prevalencia y factores de riesgo de comportamientos relacionados con suicidio en el estudio nacional de salud mental, Colombia 2003. Revista Nova, 5 (7), 84- 91.

Robinson, J., Hetrick, S. E. \& Martin, C. (2011). Preventing Suicide in Young People: Systematic Review. Australian and New Zealand Journal of Psychiatry, 45, 3-26.

Roselló, J., Berríos, M. (2004). Ideación suicida, depresión, actitudes disfuncionales, eventos de vida estresantes y autoestima en una muestra de adolescentes puertorriqueños/as. Interamerican Journal of Psychology, 38 (2), 295-302.

Rush, A.J. \& Beck, A.T. (1978). Cognitive Therapy of Depression and Suicide. American Journal of Psychotherapy, 32(2), 201-219.

Sakinofsky, I. (2007). Caring for the Suicidal Patient. Canadian Journal of Psychiatry. 52 (1), 5S-6S.

Sakinofsky, I. (2007). Treating Suicidality in Depressive Illness. Part I: Current Controversies. Canadian Journal of Psychiatry, 52 (1), 71-84.

Serrano, M. G. (2003). Suicidio: factores protectores y de riesgo en adolescentes de Mérida. Tesis inédita de maestría no publicada. México (Mérida, Yucatán): Universidad Autónoma de Yucatán.

Smith, Ph. N., Cukrowicz, K. C., Poindexter, E. K., Hobson, V. \& Cohen, L. M. (2010). The Acquired Capability for Suicide: A Comparison of Suicide Attempters, Suicide Ideators, and Non-Suicidal Controls. Depression and Anxiety, 27, 871-877.

Szumilas, M., \& Kutcher, S. (2011). Post-Suicide Intervention Programs: A Systematic Review. Canadian Journal of Public Health. Revue Canadienne De Santé Publique, 102 (1), 18-29. 
Sutton, J. M., Mineka, S., Zinbarg, R. E., Craske, M. G., Griffith, J. W., Rose, R. D. et ál. (2010). The Relationships of Personality and Cognitive Styles with Self-Reported Symptoms of Depression and Anxiety. Cognitive Therapy and Research Online DOI 10.1007/s10608-010-9336-9.
Vargas-Mendoza, J. M. (2010). Evaluación de la ideación suicida en pacientes con cáncer sometidos a quimioterapia. Centro Regional de Investigación en Psicología, 4 (1), 19-23. 